
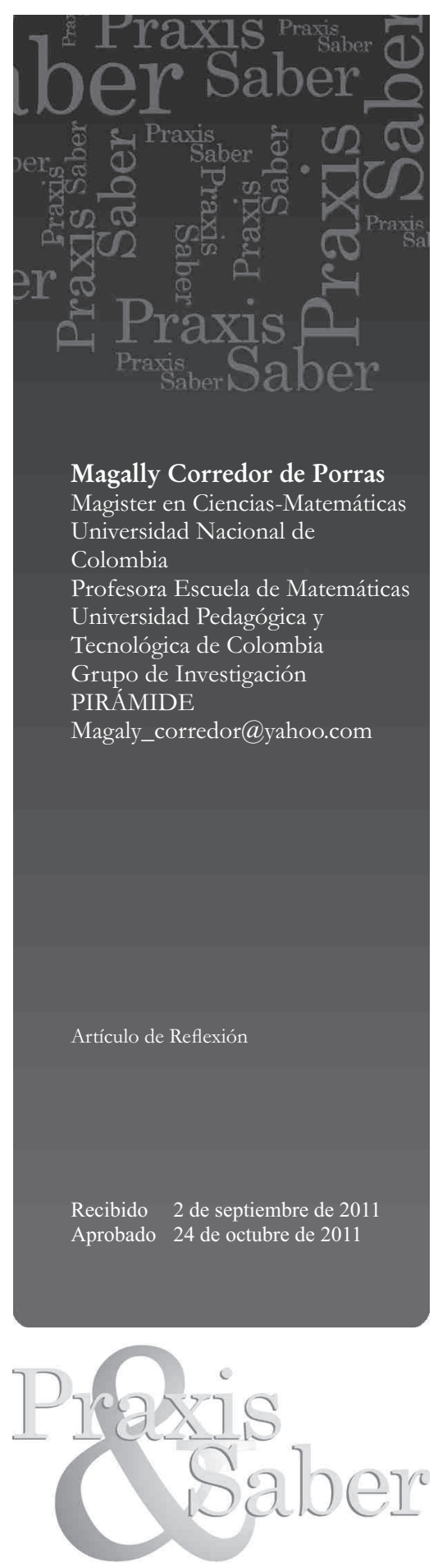

Revista de Investigación y Pedagogía Maestría en Educación. Uptc

\section{INSTRUMENTOS COGNITIVOS EN EL PENSAMIENTO MATEMÁTICO}

\section{Resumen}

Con base en la epistemología genética, de Piaget, para la cual el problema central es la construcción del pensamiento por los individuos, se examinan los instrumentos cognitivos que facilitan el progreso del pensamiento matemático antes de llegar a la formalización de los conceptos. El propósito del escrito es tomar como apoyo principal la teoría piagetiana para indagar cuáles procesos mentales se activan y cómo se va dando forma a los conceptos cuando un individuo se ve enfrentado a resolver un asunto relativo a las matemáticas. La reflexión, que se enmarca en el proyecto de investigación "Epistemología genética y matemáticas", es resultado de la revisión y complementación de algunos argumentos esgrimidos en la tesis "Mecanismos cognoscitivos comunes a la historia y la psicogénesis de la geometría". Se encuentra que el mecanismo Intra-InterTrans es un gran apoyo para comprender cómo procede la mente de los individuos, y que su estudio es vigente y tiene derivaciones útiles para la epistemología y la pedagogía de las matemáticas

Palabras clave: Mecanismo cognitivo, Generalización constructiva, Generalización completitiva, Mecanismo intra-inter-trans, Equilibración. 


\section{Cognitive Instruments in Mathematical Thinking}

\section{Summary}

Based on the Genetic Epistemology of Piaget, according to which the main problem is the construction of thoughts by individuals, this research paper examines the cognitive instruments that facilitate the progress of mathematical thinking before arriving at the organization of the concepts. The aim of this research paper is to take Piaget's theory as the main support for investigating which mental processes are activated and how they give form to concepts when an individual is faced with solving a problem relating to mathematics. This reflection, which is part of the investigative project "Genetic Epistemogy and mathematics", is the result of the revision and analysis of several arguments put forward in the thesis "Cognitive mechanisms common to the history and the psychogenesis of geometry". It is found that the Intra-Inter-Trans mechanism is of great help in understanding how the mind of individuals behaves, and that its study is valid and has useful consequences for epistemology and the pedagogy of mathematics.

Key words: cognitive mechanism, constructive generalization, completitive generalization. intra-inter-trans mechanisms, equilibration.

\section{Des Instruments Cognitifs Dans la Pensée}

\section{Résumé}

\section{MATHÉMATIQUE}

On examine les instruments cognitifs qui facilitent le progrès de la pensée mathématique avant d'arriver àla formulation des concepts ; cela, en ayant comme base l'Epistémologie génétique de Piaget, dont le problème central est la construction de la pensée par les individus. Le but de l'écrit est celui de prendre la théorie piagétienne comme soutien principal, pour indaguer quels processus mentaux s'activent et comment les concepts se façonnent quand un individu fait face à résoudre un aspect concernant les mathématiques. La réflexion, qui a comme cadre le projet de recherche “ Epistémologie génétique et mathématiques", est le résultat de la révision et de la complémentation de quelques arguments escrimés dans la thèse "Mécanismes cognitifs communs à l'histoire et la psychogenèse de la géométrie". On découvre que le mécanisme Intra-Inter-Trans est un grand 
support pour comprendre la procédure de la pensée des individus, que son étude est en vigueur et qu'il a des dérivations utiles pour l'épistémologie des mathématiques.

Mots clés: Mécanisme cognitif, Généralisation constructive, Généralisation complétive, Mécanisme intra-inter-trans, Equilibration.

\section{Instrumentos Cognitivos no Pensamento \\ Matemático}

\section{Resumo}

Baseado na Epistemologia Genética de Piaget, na qual o problema central é a construção do pensamento pelos indivíduos, examinam-se os instrumentos cognitivos que facilitam o progresso do pensamento matemático antes da formalização dos conceitos. O objetivo deste texto, baseado na teoria piagetiana indaga quais processos mentais se ativam e como se vá dando forma aos conceitos quando o individuo tem de resolver um assunto relativo à matemática. A reflexão baseada no projeto de pesquisa "Epistemologia genética e matemática" é resultado da revisão e complementação de alguns argumentos defendidos na dissertação "Mecanismos cognoscitivos comuns à história e a psicogêneses da geometria". Encontra-se que o mecanismo Intra-Inter-Trans é um grande apoio para compreender como atua a mente dos indivíduos, e o seu estudo ainda está vigente e tem derivações uteis para a epistemologia e a pedagogia da matemática.

Palavras chave: Mecanismo cognitivo, Generalização construtiva, Generalização completitiva, Mecanismo intra-inter-trans, Equilibrarão. 


\section{Introducción}

La fuente de los instrumentos de adquisición de conocimientos es la adaptación, constituida por dos procesos básicos: la asimilación y la acomodación. Con la asimilación se adopta el conocimiento como relación indisociable del sujeto y el objeto; con predominio de la actividad del sujeto, el objeto modifica en parte la asimilación por medio de la acomodación. Este carácter asimilador-acomodador del pensamiento implica una visión constructivista, pues asimilar es cercano a construir, y construcción, a estructura. En la construcción del conocimiento es importante explorar cuáles son y qué papel juegan los mecanismos de adquisición que se originan particularmente en la asimilación cognoscitiva, que es la propiedad general de las actividades del sujeto como motor activo de su propio desarrollo intelectual.

Cuando un individuo enfrenta un problema matemático intenta asimilarlo a estructuras mentales ya existentes; esto significa el intento de resolverlo mediante conocimientos que ya posee, como resultado de la asimilación; el esquema cognitivo existente se reconstruye, expandiéndose para acomodarse a la nueva situación.

Especialmente en las últimas publicaciones de Piaget es notoria la aplicación de la historia crítica de las ciencias para confirmar sus tesis; aspecto digno de rescatar, por el vasto campo de acción que señala en los estudios epistemológicos. Articular los métodos psicogenético e históricocrítico permite comprobar que una realidad no sólo es comprendida por su estado final, sino por el proceso de su elaboración; ambos métodos facilitan el análisis de los mecanismos que median la transición desde la formación inicial de las nociones hasta niveles superiores de conceptualización. La relación entre psicogénesis, o desarrollo del pensamiento en el sujeto individual, y sociogénesis, o historia evolutiva de una ciencia, muestra un paralelo en los mecanismos comunes que facilitan el movimiento intelectual de lo precientífico a lo científico. Se muestran estos instrumentos o mecanismos de paso, enfatizando en los que se refieren a la formación de conocimientos matemáticos, observando que tanto en los niveles de la inteligencia matemática, como en las etapas históricas del devenir de esta disciplina se hallan mecanismos análogos.

No se establece en modo alguno una identidad entre el desarrollo del conocimiento en el niño y el recorrido histórico de la matemática, pues son campos cognitivos distintos; es claro que el conocimiento científico acumulado no es el mismo del joven; el científico y el niño piensan diferente, pero pese a esta diferencia hay una afinidad en sus mecanismos o instrumentos de acrecentamiento del pensamiento matemático. 


\section{Los primeros instrumentos cognitivos}

Durante el periodo sensorio-motor, el constante trabajo de la asimilaciónacomodación da lugar a una organización de esquemas cada vez más elaborada y compleja, lo cual lleva a creer que los instrumentos iniciales de conocimiento no son la percepción ni el lenguaje, sino los esquemas de acción sensorio-motrices. La percepción está siempre presente en la formación del conocimiento, pero no sola; las acciones del sujeto, bien sean físicas o lógicas, agregan a los datos sensoriales un significado; la acción engloba la percepción y la supera, más aún, no es la acción aislada, sino los esquemas de acciones los que permiten elaborar el conocimiento. La raíz del pensamiento lógico es anterior al lenguaje, este es un requisito de la culminación de las estructuras lógico-matemáticas, pero no una condición suficiente (Piaget, 1970).

Un "esquema" expresa el conjunto organizado de características que permiten repetir las acciones o aplicarlas a contenidos nuevos; un esquema es una totalidad fuerte e integrada, tal que los comportamientos que induce están íntimamente relacionados (Piaget 1978). Hay esquemas innatos, como los reflejos de prensión, succión, etc., pero la mayoría no son innatos, muchos se basan en ellos y en algunas experiencias, y a su vez son causa de otras experiencias y esquemas. De una u otra forma, éstos dominan desde el principio las percepciones, y no se formalizan en conceptos o no se interiorizan en operaciones del pensamiento, sino hasta más tarde.

La primera infancia es rica en esquemas de acción: los hay de visión, prensión, tacto, succión y otros que facilitan al niño ir incorporando al campo de acción de sus esquemas más objetos variados: los objetos de chupar, los de coger, visibles, etc. Puede diferenciarlos o hacer posibles combinaciones entre éstos: por ejemplo, los objetos visibles y chupables; estas combinaciones son producto de la coordinación entre esquemas. Así, desde muy temprano hay coordinaciones entre el esquema visual y el esquema auditivo; el visual y el de succión; el visual y el de prensión, etc. Esta coordinación de esquemas da como resultado un campo de significación más amplio, donde algunos esquemas, especialmente el visual, permiten asimilar objetos a otros esquemas.

\section{Instrumentos comparativos y transformadores}

Cada esquema de acción genera correspondencias a medida que es aplicable a situaciones u objetos nuevos; la coordinación de esquemas 
genera transformaciones, ya que al producir cambios en los objetos y situaciones genera nuevas posibilidades de acción. De esta forma, los primeros instrumentos de adquisición de conocimientos matemáticos son: los instrumentos comparativos, constituidos por correspondencias, y los instrumentos transformadores, constituidos por operaciones, que actúan un poco más tarde, y aunque implican un actuar físico sobre el mundo, se realizan de manera cada vez más intelectual. Las operaciones de clasificación, por ejemplo, de seriación, de jerarquización, se componen de actividades intelectuales específicas y suponen la necesidad de la asimilación-acomodación; se basan en la interiorización y coordinación de acciones y son comparables a las nociones primigenias e intuitivas de las correspondientes operaciones en el campo de las matemáticas.

Un ejemplo de instrumento comparativo es la estrategia mediante la cual el niño puede decidir cuál de dos colecciones tiene más elementos que la otra, sin contarlos; ésto, particularmente, cuando las dos colecciones son poco numerosas. Un problema clásico que requiere un instrumento comparativo se presenta a continuación. Piaget dio a un niño ocho muñecos, que éste podría ordenar de acuerdo con la estatura; también dio ocho bastones más cortos que los muñecos; la diferencia de longitud entre pares de bastones consecutivos era menor que entre pares de muñecos adyacentes. Se pedía al pequeño que imaginara que los muñecos deben dar un paseo, y para hacerlo deben llevar el bastón más adecuado. Los más pequeños pueden lograr la correspondencia adecuada luego de varios intentos; por lo general, después de dos ordenaciones logran ordenar primero de mayor a menor los muñecos, y luego los bastones; después de ésto logran colocar el bastón más largo al muñeco más grande (Flavell, 1971). Y luego concluyen, gracias al instrumento comparativo, que las ordenaciones son equivalentes. Posteriormente, de los cuatro a los seis años, el niño emplea una estrategia distinta, comienza por el más pequeño y continúa con el menor del resto, hasta agotar la totalidad de bastones, y análogamente con los muñecos (o comenzando por el más grande); más adelante, el instrumento comparativo es mucho más fino y puede determinar la correspondencia uno a uno sin mayores dificultades.

Por su parte, las acciones interiorizadas se constituyen en instrumentos transformadores (operaciones) cuando alcanzan el nivel reversible; por ejemplo, cuando alrededor de los siete años, el niño separa (acción) por cortes una lámina y luego reúne (acción) en función de vecindades las partes que ha separado; estas dos acciones constituyen dos operaciones espaciales inversas. 


\section{Abstracciones y generalizaciones}

Más tarde, el niño emplea otros instrumentos generados en la relación asimilación-acomodación, que dependen de la naturaleza de los razonamientos, éstos son: abstracciones y generalizaciones. Puesto que se distinguen dos polos, uno de asimilación y otro de acomodación, uno como fuente de coordinaciones y otro de aplicaciones a los datos de la experiencia, se encuentran dos tipos muy diferentes de abstracción.

En primer lugar, la abstracción a partir del objeto, que extrae características, como el color, y proporciona la materia prima del conocimiento resultante de la acomodación más o menos profunda de los esquemas de asimilación. En segundo lugar, una abstracción que disgrega los aspectos particulares de la acción considerada y los mecanismos de coordinación, como unir dos o más acciones en una, o invertir las acciones, de la construcción de esquemas novedosos a través de los elementos que así se extraen de las acciones; ésto es, una abstracción que parte de la actividad del sujeto. Por estas razones la abstracción es de dos tipos: abstracción empírica y abstracción reflexiva o reflexionante; la primera obtiene la información de los objetos exteriores al sujeto, detectando las irregularidades de esos objetos, de los cuales extrae, analiza y comprueba ciertas propiedades, dejando de lado otras; la segunda se refiere a la detección de regularidades de las acciones y operaciones del sujeto. En la experiencia física actúan ambos tipos de abstracción, mientras que en la experiencia lógicomatemática, sólo la segunda.

La abstracción reflexiva o reflexionante actúa en dos sentidos inseparables: un reflejamiento, que permite pasar lo abstraído de un plano inferior a uno superior, y una reflexión, que reconstruye, reorganiza y amplía en el nuevo plano lo abstraído del plano precedente, ésto es, aquello que fue transferido por reflejamiento (Piaget y García, 1982). El reflejamiento se basa en establecer nuevas correspondencias de orden superior, y en la reflexión las correspondencias nuevas asocian las nociones transferidas con nociones nuevas que se integran a la estructura anterior, pero a la vez les permite generalizarla.

Las generalizaciones originadas en la abstracción se convierten, a su vez, en otro instrumento cognoscitivo importante; las generalizaciones también son de dos tipos: completitivas y constructivas. Las primeras son de naturaleza extensional, permitiendo el paso de "algunos" a "todos", o de casos particulares a leyes generales, o sea, consisten en un proceso completitivo que integra una estructura precedente como 
subestructura de una estructura más amplia y parcialmente nueva. La organización y reorganización de conceptos son la condición necesaria de las generalizaciones constructivas; éstas no consisten en asimilar nuevos contenidos a formas ya constituidas, sino en engendrar nuevas formas y nuevos contenidos, y, por consiguiente, nuevas organizaciones estructurales.

Un ejemplo de generalización completitiva, tomado de la historia de la geometría, es el que sigue: el grupo fundamental de la topología contiene como subgrupo el grupo de la geometría proyectiva, que contiene como subgrupo el de las afinidades, y éste contiene como subgrupo el grupo de las similitudes, y, por último, éste contiene como subgrupo el de los desplazamientos. Se puede pasar de uno de los sistemas a otro por adjunción o supresión de los invariantes característicos. Para lograr esta generalización de la geometría a nivel de la sociogénesis, se procedió del último al primero, pues se dio inicialmente la geometría euclidiana; luego, la geometría proyectiva, y posteriormente se llegó a la topología, por generalización completitiva, según la cual no se procedió por simple inclusión, ni se desembocó en una fusión trivial, sino que se dio una significación más amplia y funcional de geometría.

El siguiente es un ejemplo de generalización constructiva, tomado ahora de la psicogénesis; la noción de "grupo práctico" de los desplazamientos, es producto de una construcción debida a la coordinación de diversas actuaciones y desplazamientos; no se obtiene por una experiencia o por una simple abstracción del móvil o del movimiento del propio cuerpo, sino que se descubre en el transcurso de las experiencias o acciones ejercidas sobre el objeto, o con el cuerpo; a partir de las coordinaciones de la acción y por generalización constructiva, el niño logra no sólo el grupo práctico de desplazamientos de los objetos, sino también el grupo de sus propios desplazamientos.

\section{Instrumentos cognoscitivos y las matemáticas}

Los instrumentos mencionados hasta ahora, como herramientas cognitivas del sujeto que "hace matemáticas", nos conducen a considerar las matemáticas como ciencias extraídas de la acción y de las operaciones del sujeto, a diferencia de concebirlas como deducciones a partir de entidades dadas desde la eternidad, o "entidades ideales", como las concibe el platonismo. Así también, se diferencian las matemáticas de la concepción empírica, en la cual la primacía es del objeto que se impone 
sobre un sujeto pasivo, y según ésta, los diversos entes matemáticos son descubiertos por medio de los sentidos a partir de los objetos o de la realidad de una experiencia; de nuevo, son las acciones mismas las que intervienen en la experiencia, efectiva o mental, y la hacen posible.

En el pensamiento matemático, toda acción del sujeto está coordinada con otras; de la coordinación de acciones se extraen formas y abstracciones, y éstas, al coordinarse, a su vez dan origen por abstracción reflexiva a nuevas operaciones que son básicas para la formación de estructuras algebraicas, lógicas, geométricas o topológicas. De esta manera, el conocimiento matemático no puede considerarse preformado, innato o preexistente en una esfera ideal de la realidad, sino construido por herramientas cognoscitivas del sujeto.

Los instrumentos comparativos constituidos por correspondencias, y los instrumentos transformantes formados por operaciones, presentan en el transcurso histórico de la matemática un carácter complementario y subsidiario. La matemática griega, por ejemplo, empleó inicialmente diversas operaciones, desde la transformación de figuras hasta la manipulación de números; luego, por influjo de la filosofía más estática de la escuela eleática (Parménides), la escuela platónica menospreció el movimiento, las transformaciones y los cambios, como pertenecientes al mundo de la opinión, alejado del verdadero mundo de las ideas eternas e inmutables. Por esta actitud, la geometría griega se olvidó de las transformaciones y se reorganizó (a más tardar en Euclides, pero muy probablemente desde mucho antes) alrededor de las relaciones estáticas, como el paralelismo, la perpendicularidad, las "igualdades" o congruencias y las semejanzas.

$\mathrm{Al}$ designar las longitudes de los segmentos por medio de las letras que identifican sus extremos se hizo posible el empleo operativo de las ecuaciones, que involucran un juego de correspondencias, pero debido a la falta de una conciencia clara sobre el uso de las transformaciones, ni siquiera los algebristas del siglo XVI lograron un nivel como el que luego presentaron en el álgebra y el análisis del siglo XVII, siglo en el cual se logró una creciente relación entre las operaciones y las correspondencias.

\section{El proceso de búsqueda de razones}

Los instrumentos cognitivos que emplea el sujeto en la construcción del conocimiento dan lugar a diversos procesos que resultan importantes; 
uno de ellos, denominado por Piaget "búsqueda de razones", está presente desde el periodo sensoriomotriz hasta los niveles científicos del saber, tanto en el éxito como en el fracaso de los variados intentos que realiza el individuo en su desarrollo cognitivo. Se inicia básicamente por el deseo o la necesidad de encontrar el porqué de un evento o suceso; esta necesidad es apenas comparable con las pulsiones instintivas, y sólo una mayor comprensión de la evolución podría llegar a responder por qué el niño o adulto siente esta inquietud que lo motiva a buscar las razones de la ocurrencia de los fenómenos.

Las razones vinculan los resultados obtenidos por medio de las acciones y percepciones reguladas por las estructuras con la coordinación de esquemas y el juego interno de esas mismas estructuras; esto es, actúan como ganchos que enlazan el suceso o la acción con la red explicativa que tiene el sujeto en un momento dado. También, se puede comparar esta búsqueda de razones con el camino en el cual la persona sitúa todo suceso real entre un conjunto de posibilidades y una necesidad concebida como alternativa única; o sea, el camino en el que va eliminando las distintas posibilidades, tomando la más probable como alternativa, hasta que ésta se convierte en necesaria. El ejemplo, también clásico, de la conservación de la cantidad continua o volumen, ilustra este largo camino:

Se presentan dos vasos iguales, 1 y 2 , cada uno con igual cantidad de agua; dos vasos más, 3 y 4, diferentes entre sí y a los primeros, y una serie de cuatro vasos más pequeños, 5 (ver figura 1). Luego de que el niño confirma que, en efecto, 1 y 2 tienen igual cantidad de agua, se le pide que vierta el líquido de 2 en 3 , y posteriormente en 4 ; se le pregunta si los vasos 1 y 3 contienen igual cantidad de agua; si responde afirmativamente, se le pregunta por qué. El líquido vuelve de 3 a 2 , y se le vuelve a preguntar si 1 y 2 contienen igual cantidad. Se repite la actividad detallada, esta vez con el recipiente 4. Posteriormente se vierte el fluido de 1 o 2 en la serie de vasos 5 , y se le interroga en el mismo sentido anterior; si el niño sigue afirmando en cada caso que la cantidad de agua de 2 vertida en las diferentes vasijas es igual al de 1 , entonces ha comprendido la conservación de la cantidad continua; si no admite esta equivalencia, el individuo todavía no ha logrado esta conservación. 


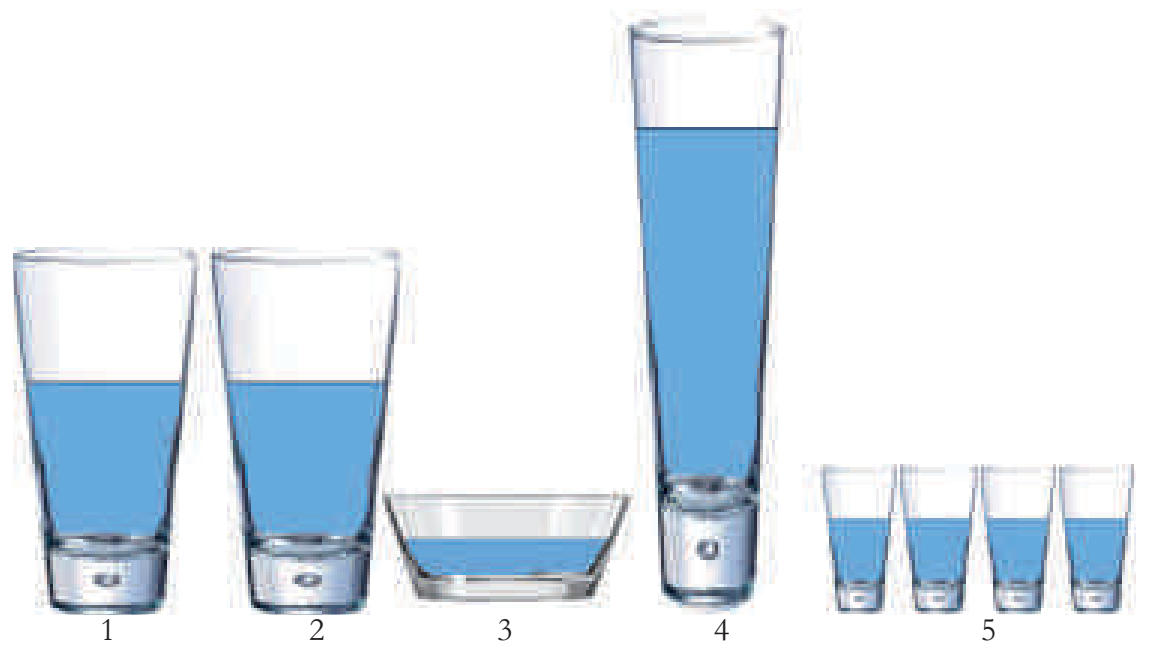

Figura 1. Ilustración de la experiencia para proceso de búsqueda de razones.

Con niños entre cuatro a cinco años se tiene éxito en la igualdad de 1 y 2; al verter 2 en 3 ya no mantienen la igualdad, manifiestan que 1 tienen más que 3, porque la altura de la columna de agua es mayor; en esta justificación, el niño se fija solamente en la altura; para él, a iguales alturas, iguales cantidades de agua, y cuando las alturas son diferentes hay menos líquido en la menor; es el caso de 3 con respecto a 1. Los niños de cinco a seis años vacilan; algunas veces tienen en cuenta la altura y otras la anchura; unas veces manifiestan que la más alta contiene más, y otras que la más ancha; a pesar de ésto, la diferencia con el sujeto de la etapa anterior es que no sólo tiene en cuenta la altura, sino también la anchura. Desde los seis años se es capaz de mantener la conservación.

En el primer caso, el sujeto se enfrenta al problema fijándose en una sola dimensión, por ejemplo la altura; es la estrategia más plausible para él, pero también la que lo conduce a una valoración inadecuada de la situación, pues se basa en aspectos estáticos del medioambiente y no en la transformación que se produce; cuando tiene en cuenta los dos factores, altura y anchura, aunque en forma aislada, emplea una segunda estrategia que enfoca a la vez, o alternadamente, las dos dimensiones, alto y ancho. Sin embargo, a la postre tiene en cuenta sólo la dimensión más notoria: en el caso de 3, la anchura, y en el caso 4, la altura. Esta segunda estrategia es muy inestable todavía. Una tercera estrategia, que oscila entre las dos dimensiones, le hace ver que una de ellas no cambia sin que se produzca un cambio inverso en la otra; a más altura, menos anchura y 
viceversa; cuando logra combinar las dos dimensiones y relacionarlas con la transformación es cuando logra el éxito. Aunque al comienzo la última estrategia no era la más probable, ha llegado a serlo como resultado de las estrategias previas. Experimentando los conflictos y eliminando poco a poco las posibilidades, el niño desarrolla una estrategia con mayor probabilidad de éxito, que finalmente lo lleva a evaluarla como aquella que necesariamente le satisface y le permite dar una explicación segura en todos los casos.

El enriquecimiento que el sujeto adquiere situándose en lo posible y lo necesario lo conduce a establecer un doble movimiento de asimilación a acomodación, y viceversa, que en últimas lo lleva a establecer un equilibrio dinámico entre éstas; sin embargo, hay que recordar que ni lo posible ni lo necesario son observables: ambos son producto de la actividad del sujeto dentro del proceso.

Ningún sujeto que ha llegado a un cierto nivel de conocimiento se contenta con comprobar o descubrir; en cada etapa, los sujetos cognoscentes buscan llegar a las razones de aquello que han encontrado; esto equivale a buscar, en cada caso, bajo la generalidad de las relaciones, causas y conexiones establecidas, la necesidad interior de que ese caso ocurra, pues el sujeto tiene la tendencia a reconocer como válida una construcción solo en la medida en que ella se hace necesaria en virtud de razones explícitas. Esto a su vez explica el porqué del progreso intelectual, puesto que el individuo mismo es quien genera las diversas formas de una necesidad progresiva, cuya conquista se logra únicamente por fases.

Un suceso observado fácticamente no puede considerarse posible sino sobre el telón de fondo de una combinatoria activa con la que el sujeto genera ese y los demás posibles, y no puede considerarse necesario sino sobre ese mismo trasfondo combinatorio y bajo la luz de un juego deductivo teórico que excluye la ocurrencia de todos los demás sucesos inicialmente considerados posibles.

\section{Diferenciaciones e integraciones}

Otro proceso derivado de los instrumentos cognitivos, igualmente importante, es el doble proceso de diferenciaciones e integraciones. El individuo, inicialmente, no diferencia los seres vivos de los no vivientes, muestra un alto grado de indiferenciación de las variables, las confunde con los factores que intervienen en el movimiento, y establece correspondencias ilegítimas entre cuerpos inertes y vivientes. Posteriormente, en otro nivel, 
el niño se desprende del motor interno y acoge la idea de acción global, es decir, la transmisión del movimiento a través de intermediarios inmóviles; se lleva a cabo entonces la primera diferenciación entre los elementos vivientes y los no vivientes; es así como el niño elimina la actividad interna de los móviles. En un tercer nivel se invoca un impulso como causa del movimiento y se diferencia entre velocidad e impulso y entre impulso y fuerza. En el cuarto nivel el movimiento es descrito como un aspecto dependiente de si hay o no fuerzas presentes que causen aceleración; se descubre empíricamente la aceleración, pero sin diferenciarla claramente de la velocidad (Piaget y García, 1982).

El estado inicial de indiferenciación expresa una dificultad de imaginar otras posibilidades diferentes a aquellas que son aceptadas en un momento dado. Es fundamental esa indiferenciación inicial entre lo real, lo probable y lo necesario. La fase siguiente está caracterizada por las primeras diferenciaciones, donde encontramos apertura hacia nuevas posibilidades y construcción de otras necesidades, concluyendo con una integración de lo nuevo a lo viejo, enriqueciendo las nociones y las estructuras previamente desarrolladas.

\section{Toma de conciencia}

Este instrumento, como proceso que posibilita la significación, es igualmente corresponsable de la superación hacia formas nuevas de conocimiento. Es frecuente que tanto el niño como el científico atraviesen por periodos donde ciertas operaciones y nociones desempeñan solamente un papel instrumental, sin toma de conciencia suficiente acerca de lo que se hace o se piensa, de allí pasan a fases posteriores donde las mismas operaciones y nociones son tematizadas y dan lugar a nuevas operaciones, conceptos y teorías (Piaget y García, 1982).

En la evolución de las matemáticas, tanto en el campo histórico como en el psicológico, la tematización puede considerarse una conceptualización rigurosa de las entidades matemáticas ya construidas progresivamente apenas como instrumentos, y es anterior a la axiomatización; se trata de una conceptualización que se basa en un "saber hacer" o en acciones antes no tematizadas ni conscientes, ésto es, carentes de significado en un contexto o momento determinado. Puede decirse que las tematizaciones dan lugar al prolongamiento de las abstracciones reflexionantes. Se observa en el siguiente ejemplo de la sociogénesis de las matemáticas modernas:

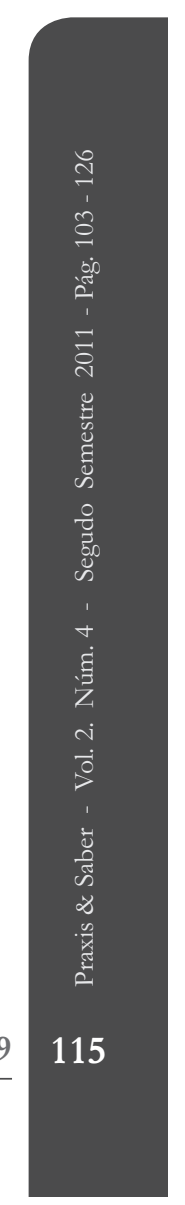


Cuando Gauss estudió las funciones de dos variables de la forma $\mathrm{f}(\mathrm{x}, \mathrm{y})=\mathrm{ax}^{2}+2 \mathrm{bxy}+\mathrm{cy}^{2}$, con a,b,c pertenecientes a $\mathrm{Z}$, a las que llamó "formas", definió la composición de éstas y obtuvo diversas propiedades que no podían deducirse directamente de las operaciones entre números. Estas propiedades hoy en día se pueden traducir en el lenguaje de la teoría de grupos; una colección de clases de equivalencia de formas cuadráticas, con una ley de composición como la que definió Gauss, es un grupo abeliano cuyo elemento unitario es la clase que él denominó "clase principal". Este brillante matemático, al igual que otros célebres matemáticos de siglo XIX, llegó muy cerca de la teoría de grupos, pero no la constituyó, quizá porque su propósito consistió sólo en transformar funciones y en encontrar las relaciones que permanecen estables; pero hay una larga senda para pasar de una colección de transformaciones a una estructura total, dentro de la cual aquellas resultan ser variaciones intrínsecas producidas por la actuación de la ley de composición interna sobre uno o más generadores.

Cambiando la pregunta inicial: ¿qué clase de números determinan las propiedades de los polinomios o de sus ceros?, por la pregunta: ¿qué propiedades tienen los números que intervienen en tales consideraciones?, fue como esas propiedades fueron apareciendo, y se vio que no constituían propiedades de los números, sino que además de éstos, muchos otros conjuntos tenían las mismas propiedades; de esta manera se fue tomando conciencia de que las propiedades comunes de tales conjuntos no definen un ente específico dentro de los objetos matemáticos, sino una estructura común a muchos de tales entes. Es así como Galois, al tomar conciencia de lo que se tematizaría posteriormente como la estructura de grupo (primera estructura algebraica completamente tematizada en matemáticas hacia 1882), dio comienzo al estudio de las estructuras; se ve que esta toma de conciencia y tematización permitió abordar un nuevo campo de estudio, en este caso el álgebra abstracta.

El proceso de toma de conciencia y tematización también está presente en psicogénesis. Se pide a un grupo de niños que hagan girar en círculos una piedra amarrada a una cuerda, y que luego la suelten, con el fin de hacerla caer en una caja situada frente a ellos. En forma rápida aprenden a lanzar la piedra desde las posiciones tangenciales precisas; ésto es, al imaginar el círculo que describe la piedra como un reloj en el que las doce están frente a la caja, los niños sueltan la piedra en la posición de las tres o de las nueve, según el sentido del giro; pero al preguntarles por la posición en la cual han liberado el objeto, creen haberlo hecho en frente de la caja, o sea, la posición de las doce; hay acá una ausencia de conciencia sobre 
lo que se hace, a la vez que una falsa necesidad de creer que el objeto no llega a la caja si no es lanzado desde un punto que, situado en frente, se una en línea recta con el blanco, lo que prueba que hay un camino largo por recorrer entre el empleo espontáneo e inconsciente de estructuras y la toma de conciencia de tal uso.

\section{Mecanismos de transición en la sociogénesis y en la psico- génesis}

El presente análisis de los mecanismos comunes del progreso intelectual tanto en la sociogénesis como en la psicogénesis ha enfatizado en los mecanismos de la construcción y del progreso del saber, más que ocuparse del contenido de las nociones mismas; lo fundamental no es preguntar acerca de qué piensa el sujeto, sino indagar cómo ha procedido para adquirir tal o cual noción. La analogía entre los mecanismos permite concebir las transformaciones del conocimiento en forma semejante en los dos campos. Las reglas de transición que impulsan a superar continuamente lo que ha adquirido están vinculadas con la asimilación de lo nuevo a estructuras precedentes y con la acomodación de estas estructuras a las nuevas adquisiciones hechas por los individuos.

El mecanismo de equilibración consiste en lograr una coordinación equitativa entre la asimilación y la acomodación, es el motor del cambio y de la transición en el proceso general del conocimiento, dando lugar a estadios de equilibrio sucesivos; cada uno se refiere a un sistema estable de relaciones entre el sujeto y el objeto, por tanto, a una relación armónica entre la asimilación y la acomodación. La equilibración connota una especie de complementación mutua, una fase homeostática en la cual las acomodaciones a la realidad son controladas mediante un proceso asimilativo que puede organizar y orientar las acomodaciones.

Es necesario disipar un equívoco que suele ocasionar la palabra "equilibrio". Un sistema cognoscitivo que ha alcanzado el equilibrio no se halla propiamente en reposo, sino que interactúa con el medioambiente; el sistema trata los acontecimientos ambientales en función de sus estructuras (asimilación) y puede modificarse en consonancia con las exigencias ambientales (acomodación). Cuando hay equilibrio, el sistema cognoscitivo no necesita distorsionar los acontecimientos para asimilarlos, ni necesita cambiar demasiado sus estructuras para acomodarlas a los nuevos acontecimientos. En consecuencia, el equilibrio implica actividad, apertura y una fase de relativa armonía con el medioambiente; es un equilibrio dinámico. 
Volviendo al ejemplo de la conservación del volumen, el niño de la fase preoperatoria es capaz de manejar solo una reversibilidad empírica, pues al verter de nuevo el líquido del vaso 3 en 2, afirma que hay igual cantidad que antes; pero este tipo de reversibilidad no le permite aseverar si existe más o menos agua en 3. Acá, el sujeto ve las acciones de verter 2 a 3 , y 3 a 2 como acciones independientes. Con más edad, concluye que verter 3 a 2 invierte la acción de verter 2 a 3 , y hay conciencia de que la acción se realiza en otra dirección; entonces, maneja la reversibilidad. Al alcanzar la conservación ha adquirido un buen nivel de equilibrio, al cual accede solo con la estrategia final.

El mecanismo de equilibración es dinámico; comienza transformando una estructura bajo influencias de nuevas situaciones, y a la vez conduce a construir una nueva estructura; aunque comienza por desequilibrios parciales, tarde o temprano consigue el restablecimiento de una nueva forma de equilibrio que corresponde a una estructura nueva. Hay dinamismo, pues no hay una estructura definitiva que sea consumación de toda construcción. Ninguna estructura es radicalmente nueva, sino que cada una está limitada a generalizar alguna forma de acción abstraída de la anterior. Además, el equilibrio es reversible, es decir, tiene la propiedad de invertir las acciones; una acción realizada en forma aislada y sin reversibilidad conduce a relaciones sin equilibrio (Flavell, 1971).

Los estados de equilibrio presentan propiedades: campo de aplicación, movilidad y estabilidad (Flavell, 1971). El campo de aplicación se relaciona con los objetos y con sus propiedades; por ejemplo, en la percepción visual, el campo de aplicación es la imagen visual, es decir, los objetos que una persona puede captar en una sola mirada. En la clasificación, el campo es bastante amplio e implica la lista de elementos de la clase concreta; esta lista puede, por supuesto, contener una cierta variedad de objetos. Cuanto mayor es el campo de aplicación, más fuerte es el equilibrio.

La movilidad se define en términos de distancias temporales y especiales que separan a la persona y los elementos del campo de aplicación; si son grandes estas distancias, más móvil es el equilibrio o más flexibles son las operaciones mentales necesarias para cubrir estas distancias. En el campo visual las distancias espaciales y temporales son pequeñas y exigen un número relativamente limitado de actividades mentales. Clasificar exige la representación de objetos que no se hallan presentes, y, por lo tanto, las distancias espaciales y temporales entre los elementos son ilimitadas; en el caso de los objetos inmediatamente presentes, exige una variedad de operaciones mentales, por ejemplo, alineaciones (ejemplo de muñecos y bastones), inclusiones, etc. 
La estabilidad se relaciona con la capacidad de compensar mediante acciones u operaciones mentales los cambios en los elementos sin trastornar la estructura total previa; ésto es, cancelar perturbaciones que tienden a alterar el estado de equilibrio existente. En la percepción visual el equilibrio es parcial y limitado; si se introducen nuevos elementos en el campo visual, se cambia la percepción; es el caso de las ilusiones ópticas, el caso más sencillo es de la figura 2.
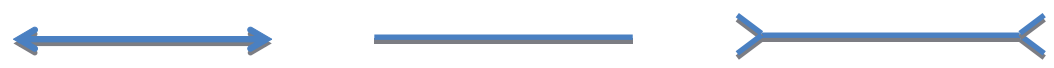

Figura 2. Propiedad de estabilidad del mecanismo de equilibrio.

La introducción de nuevos elementos en la clasificación no destruye el sistema; por ejemplo, si el niño clasifica los útiles escolares en lápices y no lápices, al descubrir un cuaderno lo clasifica como no lápiz, es decir, al clasificar se pueden tomar en cuenta elementos adicionados sin distorsión alguna, mientras que el perceptivo se afecta e inclusive se distorsiona por la adición de nuevos elementos.

Sin embargo, hay tipos de cognición que no muestran equilibrio: los juegos y la imitación. En el juego (inclusive formas de fantasías) el propósito es amoldar la realidad al capricho del jugador; se asimila la realidad a esquemas, sin dar importancia a la acomodación a esa realidad; por tanto, hay primacía de la asimilación sobre la acomodación, y se adapta la realidad al yo. En la imitación se toma cuenta exacta de los detalles de la realidad que se imita, y se amoldan los esquemas propios a esos detalles; la acomodación prima sobre la asimilación, y adaptamos el yo a la realidad.

\section{El mecanismo intra-inter-trans}

El mecanismo intra-inter-trans conduce el conocimiento de lo intraobjetal a lo interobjetal y, más tarde, a lo transobjetal. Lo intraobjetal, básicamente, es un análisis de los objetos o eventos con descubrimiento de propiedades intrínsecas que obedecen a explicaciones todavía muy particulares y locales. Lo interobjetal busca explicaciones a las relaciones entre objetos o eventos desde un punto de vista más amplio, con lo que se impulsa al hallazgo de las transformaciones, las que, junto con las relaciones, constituyen lo característico de lo interobjetal. Lo transobjetal establece vínculos entre las transacciones, los cuales implican la construcción de estructuras; las estructuras, por su parte, son lo propio de lo transobjetal. A manera de notación se empleará: Ta, para el periodo Intra; Tr, para el 
periodo Inter, y Ts, para el periodo Trans; así, se escribirá la triada IntraInter-Trans como TaTrTs. (Piaget y García 1982).

La sucesión de los periodos Intra-Inter-Trans está orientada según un orden necesario, ya que en el Trans la totalidad de las transformaciones con sus vínculos y propiedades supone la formación de algunas o todas esas transformaciones en el Inter, y éstas, a su vez, implican conocer las características y propiedades intrínsecas, encontradas ya en la etapa Intra. Este orden sucesivo y necesario impulsa superaciones, llamadas por Piaget "rebasamientos", en el sentido del rebasamiento de una estructura de conocimiento por una más amplia, donde lo que ha sido rebasado está presente en lo que lo ha rebasado. Los rebasamientos están orientados del Inter con respecto al Intra, y del Trans con respecto al Inter.

La triada TaTrTs induce la construcción de operaciones sobre otras, puesto que en el Ta se analizan casos particulares sin nexos entre sí o con una vinculación insuficiente; en el Tr la comparación en los diferentes casos particulares conduce a la construcción de transformaciones, en virtud de que pone en evidencia tanto las diferencias como las analogías, y establece correspondencias, y en el Ts las correspondencias derivadas del $\operatorname{Tr}$ son sistematizadas en totalidades hasta entonces inaccesibles. Es también por esta razón por la que la secuencia TaTrTs describe el aspecto dinámico de los rebasamientos.

Un ejemplo extraído del desarrollo histórico del álgebra (Piaget y García 1982): se inaugura el desarrollo del álgebra con el interés de resolver ecuaciones determinadas, empleando un método puramente empírico basado en el tanteo; cada ecuación se estudió en forma particular y aislada, dando a cada una un tratamiento individualizado; en este comienzo las operaciones algebraicas se particularizan e individualizan de acuerdo con las circunstancias y con la naturaleza de la ecuación; corresponde a un periodo Intraoperacional o simplemente Intra $(\mathrm{Ta})$. Hacia el siglo XVIII, el interés se desvía ligeramente a indagar por las condiciones que median la existencia o inexistencia de soluciones para ecuaciones dadas. Para el caso de existencia de soluciones, se buscaron métodos generales de solución, aplicables a diversas ecuaciones del mismo grado, distinguiendo métodos distintos para tipos distintos, pero procurando integrar ecuaciones aparentemente muy distintas dentro del mismo tipo; este análisis abrió la puerta a las transformaciones de ecuaciones no solubles en ecuaciones resolubles, aspecto que fue estudiado entre otros por Lagrange y Gauss; este periodo, por sus características, es interoperacional, o simplemente Inter $(\mathrm{Tr})$. Finalmente, el auge y el desarrollo de la teoría de grupos, los 
cuales abren paso al análisis de ésta y otras estructuras algebraicas, como los anillos y los cuerpos, constituyen un nuevo período, en el cual se trabajan a un nivel de abstracción y de tematización más alto las operaciones y los axiomas sobre éstas; podemos llamar a este periodo transoperacional, o simplemente Trans (Ts).

En línea paralela, para el desarrollo psicogenético del álgebra se puede identificar la sucesión de periodos TaTrTs. Se da un primer periodo, que oscila entre los cuatro y siete años de edad; el niño se mantiene sujeto a una acción correcta que repite, pero que no relaciona con las causas ni con las consecuencias que le permitan ampliar el alcance de la preoperación; su comprensión y esfuerzo se centran en la naturaleza y las propiedades de la preoperación considerada aisladamente; es el caso de la experiencia en que se pide a niños que añadan, una tras otra, tapas a dos bolsas, una transparente y otra opaca, echando con la mano izquierda una tapa a la bolsa transparente, y con la derecha una a la otra bolsa, en forma simultánea; para un número pequeño de agregaciones el niño conserva la igualdad del número de tapas de ambas bolsas, pero si se le pide que generalice esta consecuencia al caso en que la operación se repite por un lapso más largo, se niega a contestar, o responde que no se puede decidir hasta cuando se hayan contado las tapas; este periodo preoperativo reúne rasgos propios de un periodo Intra. De los siete a los diez años, después de comprender una operación, el sujeto la emplea como punto de partida para deducir otras operaciones o para coordinarla con otras más o menos análogas, lo que puede llevarlo a involucrar transformaciones; así, en el ejemplo anterior, los niños de este nuevo periodo apuntan rápidamente a la conservación de la igualdad del número de tapas, aún para periodos de tiempo mucho más amplios.

La negación y la reciprocidad son dos transformaciones generales; la primera como transformación de los conjuntos de clase, y la segunda como transformación de los agrupamientos de relaciones; estas transformaciones son dos formas posibles de reversibilidad; el hecho de que estén presentes en este periodo marca el avance al periodo Interoperacional con referencia al Intraoperacional.

Volviendo al ejemplo de la conservación de la cantidad continua, el sujeto de más de seis años brinda varias razones cuando se le pregunta por qué permanece la cantidad de agua tras haberla vertido; una es la negación: si el líquido de 3 volviera a su contenido original, 2, entonces los dos vasos iniciales, 1 y 2 , contendrían igual cantidad de líquido (devuelve la acción): una segunda razón es el argumento de identidad: permanece 
igual la cantidad porque se trata de la misma agua; un tercer argumento, de reciprocidad: se mantiene la igualdad, pues no se ha añadido ni quitado nada; el sujeto invoca una compensación, pues 3 es más baja que 1, pero lo que 3 perdió en altura quedó compensado al ganar en anchura (en 4), por lo tanto, la cantidad de agua de 3 es igual a la de 1. El individuo presta atención a ambas dimensiones: un incremento destruye al otro, y por ello la reciprocidad es una forma de reversibilidad. También, invertir el acto del vertido (negación) reconoce que el estado final es la disposición inicial 1 y 2 , y que estos cambios 2 a 3 , y 3 a 2 pueden ser anulados; esa es otra forma de reversibilidad llamada correlativización. Ambas se dan cuando el individuo centra su atención en la transformación y no en puntos de referencia estáticos. Conviene fijarse cómo las operaciones de reversibilidad se emplean de manera independiente una de otra.

El periodo de los once a los catorce años, y de allí en adelante, corresponde a un periodo Trans, en el cual, además de involucrar las transformaciones, se construyen estructuras relativas a las acciones que permanecen interiorizadas en forma de operaciones, y las negaciones y reciprocidades se coordinan entre sí para lograr una estructura del grupo. Si $\mathrm{p} \Rightarrow$ es la operación binaria sobre la que se va a actuar, sean:

I: operación identidad

$\mathrm{N}$ : operación negación

$\mathrm{R}$ : reciprocación

C: operación correlativización

$$
\begin{aligned}
& \mathrm{I}(\mathrm{p} \Rightarrow \mathrm{q})=(\mathrm{pq})=(\sim \mathrm{p} \vee \mathrm{q}) \\
& \mathrm{N}(\mathrm{p} \Rightarrow \mathrm{q})=(\mathrm{p} \wedge \sim \mathrm{q})=\sim(\mathrm{p} \Rightarrow \mathrm{q}) \\
& \mathrm{R}(\mathrm{p} \Rightarrow \mathrm{q})=(\mathrm{q} \Rightarrow \mathrm{p})=(\sim \mathrm{p} \Rightarrow \sim \mathrm{q})=(\mathrm{p} \vee \sim \mathrm{q}) \\
& \mathrm{C}(\mathrm{p} \Rightarrow \mathrm{q})=(\sim \mathrm{p} \wedge \mathrm{q})=\sim(\sim \mathrm{p} \Rightarrow \sim \mathrm{q})
\end{aligned}
$$

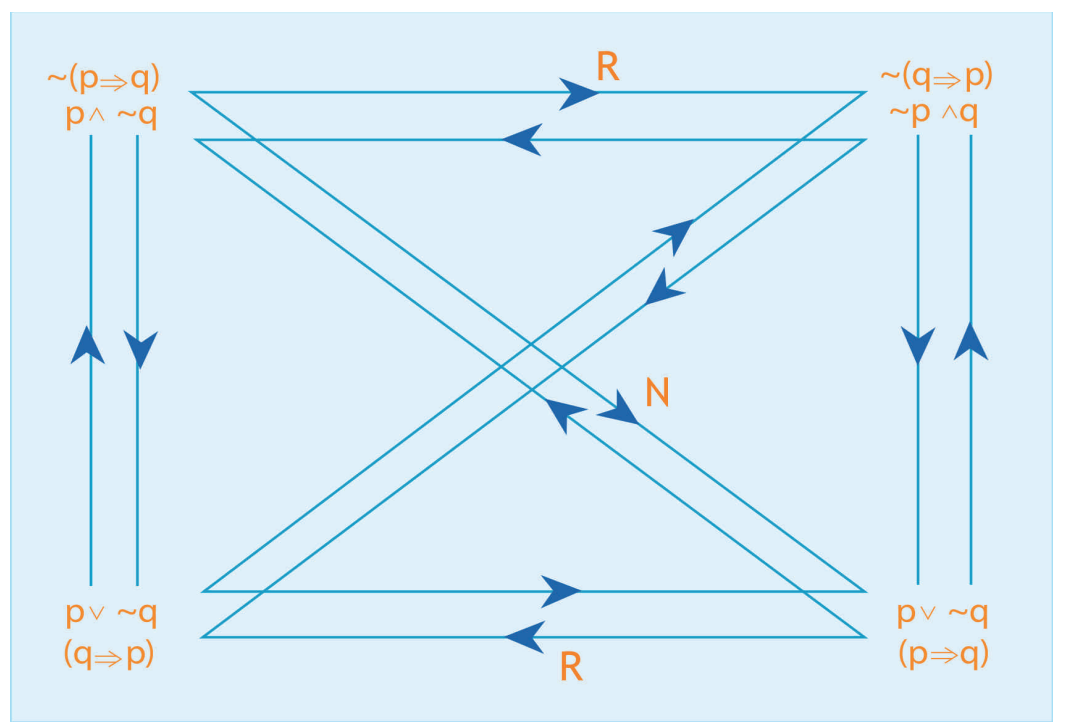




$$
\begin{array}{llll}
\text { NoR }=\mathrm{C} & \text { NoC }=\mathrm{R} & \mathrm{CoR}=\mathrm{N} & \text { NoCoR }=\mathrm{I} \\
\mathrm{R} \text { o }=\mathrm{C} & \mathrm{CoN}=\mathrm{R} & \mathrm{RoC}=\mathrm{N} &
\end{array}
$$

\begin{tabular}{|c|c|c|c|c|}
\hline $\mathrm{O}$ & $\mathrm{I}$ & $\mathrm{N}$ & $\mathrm{R}$ & $\mathrm{C}$ \\
\hline $\mathrm{I}$ & $\mathrm{I}$ & $\mathrm{N}$ & $\mathrm{R}$ & $\mathrm{C}$ \\
\hline $\mathrm{N}$ & $\mathrm{N}$ & $\mathrm{I}$ & $\mathrm{C}$ & $\mathrm{R}$ \\
\hline $\mathrm{R}$ & $\mathrm{R}$ & $\mathrm{C}$ & $\mathrm{I}$ & $\mathrm{N}$ \\
\hline $\mathrm{C}$ & $\mathrm{C}$ & $\mathrm{R}$ & $\mathrm{N}$ & $\mathrm{I}$ \\
\hline
\end{tabular}

Figura 3. Esquema de operaciones del grupo $\{\mathrm{I}, \mathrm{N}, \mathrm{R}, \mathrm{C}\}$

El conjunto $L=\{\mathrm{I}, \mathrm{N}, \mathrm{R}, \mathrm{C}\}$ con la operación "o" constituye un grupo isomorfo al grupo de Klein $\mathrm{K}_{4}:\langle L$, o $>$. Esta estructura es empleada por el sujeto aún cuando no la tematice, ni tenga conciencia de su uso (Vasco, 1989). Se trata de un modelo lógico que intenta describir la estructura de las actividades mentales del adolescente, por cuanto muchos adolescentes no conocen la lógica proposicional, ni las operaciones de grupo.

Puesto que todo nivel obedece a fases de formación, es posible distinguir subetapas. Sorprende el hecho de que cada etapa a veces repite en sus propias fases el mecanismo total, ésto es, la sucesión TaTrTs. Por ejemplo, si bien es cierto que la estructura de grupo es una construcción propia del periodo transoperacional, es posible distinguir en su proceso de tematización, a su vez, tres subetapas: una trans-intra, en la cual se trabajaron las permutaciones, que no provenían de una construcción debida al grupo mismo, sino de la construcción de arreglos al tanteo; esta subetapa se cumple del siglo XIV al siglo XVIII en Europa. Otra subetapa, trans-inter, caracterizada por los grupos de transformaciones, en los cuales las transformaciones (llamadas "sustituciones") ya forman parte del grupo; esta subetapa se cumple en el siglo XIX, de Galois a Jordan. Llega finalmente una subetapa trans-trans, correspondiente a la elaboración de la noción abstracta de grupo, aplicada sobre cualquier conjunto y cualquier operación; esta última subetapa está caracterizada por la conceptualización explícita y la aplicación intencional de la estructura, y solo se logra a fines del siglo XIX.

A partir del siglo XIX se distingue la acción de sustituir unos símbolos por otros, llamada "sustitución", del resultado de esa acción, como arreglo estático de símbolos llamado "permutación"; en términos actuales, el elemento del "grupo de permutación": $\mathrm{S}_{\mathrm{n}}$, no es pues la permutación misma, sino la sustitución. Piaget habla entonces de la operación de sustitución, pues es la función unaria, y de la operación del grupo: la composición, que es una función binaria. 
El mecanismo TaTrTs tiene dos direcciones en las construcciones que de él se derivan: la dirección retroactiva y la dirección proactiva (Piaget y García, 1982). Retroactiva, pues lo adquirido en un nivel determinado enriquece posteriormente lo establecido en niveles anteriores, y proactiva, en la medida en que hay ampliación de dominios en periodos sucesivos. Suponiendo las etapas I, II y III como sucesivas en una línea de desarrollo, cada una con sus tres subetapas, por ejemplo, para la etapa II, correspondiente al inter del desarrollo, en el momento 1: (Intra de II) se dan reinterpretaciones de la etapa I, en el sentido en que retroactivamente se la subyuga a operaciones de tipo II, o sea, de tipo inter, y en el momento 2: los conceptos y elementos de 1 en II intervienen en operaciones de tipo Inter en 2 , y así sucesivamente.

La dirección proactiva de la triada TaTrTs se encuentra tanto en las etapas como en las subetapas, y la dirección retroactiva actúa sobre las construcciones y adquisiciones anteriores por medio de las reorganizaciones. Es acá donde la organización pone en marcha la generalización constructiva como instrumento cognoscitivo.

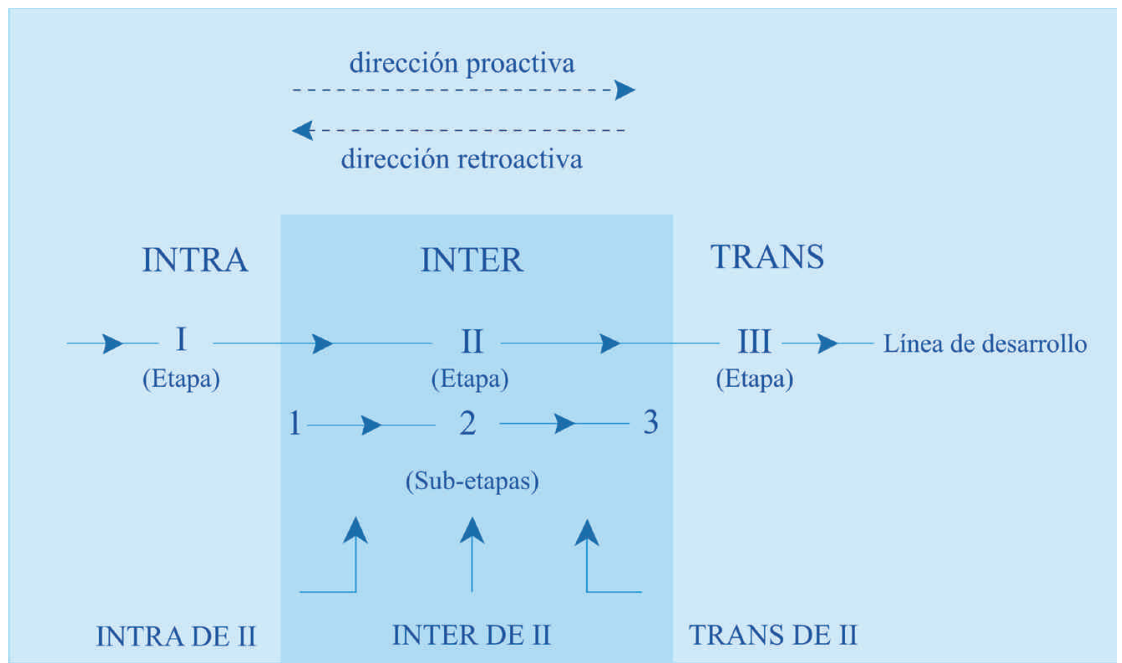

Figura 4. Línea de desarrollo y direcciones de construcción del mecanismo TaTrTs

A manera de ejemplo: en un periodo Intra el sujeto maneja diversos casos de conmutatividad de la adición, aisladamente; observa a base de conteos que es lo mismo $3+2$ que $2+3$; puede aumentar el número y deducir el resultado análogo, por ejemplo, con $12+3=3+12$; por otra parte, hace observaciones similares para el caso de la multiplicación 2 
$\times 3=3 \times 2$, luego $12 \times 4=4 \times 12$, sin que establezca comparaciones entre la conmutatividad de las dos operaciones, o sea, mantiene esta propiedad en un nivel particularizado. En un periodo Inter, el individuo generaliza el comportamiento, conceptualiza la conmutatividad de la adición y conceptualiza la conmutatividad de la multiplicación; hay acá una dirección proactiva del mecanismo, en el sentido de que se amplía el dominio del concepto de casos o ejemplos particulares a la ley, y en tanto que se comparan los dos casos de conmutatividad que hasta entonces habían permanecido aislados, gracias a la generalización constructiva. Una proposición como: "tanto la suma + como la multiplicación x son conmutativas" es claramente del periodo Inter. En un periodo Trans el sujeto establece vínculos entre éstas y otras operaciones que manifiestan la misma propiedad, y entonces tematiza la conmutatividad en sí misma, haciendo abstracción de la operación y de la naturaleza de los elementos sobre la cual se aplica; infiere el caso más general: Si $\forall \mathrm{x}, \forall \mathrm{y}, \mathrm{x} * \mathrm{y}=$ $\mathrm{y} * \mathrm{x}$, entonces la operación $*$, cualquiera que sea, es una operación conmutativa. Nótese que el "sujeto gramatical" de esta proposición no son las variables (para elementos) $\mathrm{x}$, y, sino la variable (para operaciones) *. Se trata, pues, de una proposición abierta que se puede simbolizar Con $m(*)$, en la cual no figuran los elementos, sino sólo la operación genérica *, y por lo tanto se trata claramente de una proposición del periodo Trans.

También la dirección retroactiva está presente, pues el sujeto, a partir de reorganizaciones y reinterpretaciones de lo adquirido en periodos anteriores, infiere, en este ejemplo, el caso más general, así amplía el dominio de validez de la propiedad; como también en la medida en que los casos de conmutatividad de la adición y de la multiplicación de números, primero naturales, luego racionales y por último reales y complejos, enriquecen lo establecido en el nivel Trans. Una manifestación llamativa de la dirección retroactiva de la etapa Trans es para este caso la convicción implícita que tienen muchos matemáticos de que la conmutatividad es una propiedad "natural" y "frecuente" de las operaciones binarias, cuando en realidad las composiciones solo excepcionalmente son conmutativas.

\section{Referencias}

CORREDOR, Magaly (1993). Mecanismos cognoscitivos comunes a la bistoria y la psicogénesis de la geometría. Tesis de maestría en Ciencias-matemáticas, Universidad Nacional, Director: Carlos E. Vasco. Bogotá. 
Flavelu, John (1971). La psicología evolutiva de Jean Piaget. Buenos Aires: Paidós.

Ginsburg, Herbert y Opper, Sylvia (1985). Piaget y la teoría del desarrollo intelectual. México: Calipso.

Piaget, Jean (1970). Naturaleza y métodos de la epistemología. Buenos Aires: Proteo.

Piaget, Jean (1970). Psicología y epistemología. Buenos Aires: Emecé.

Piaget, Jean (1978). Introducción a la epistemología genética, 1. El pensamiento matemático. 2 ed. Buenos Aires: Paidós.

Piaget, Jean y García, Rolando (1982). Psicogénesis e historia de la ciencia. México: Siglo XXI.

VAsco, Carlos Eduardo (1989). "Dos grupos piagetianos en la lógica elemental". Revista de la Academia Colombiana de Ciencias Exactas, Físicas y Naturales. XVII(64): 29-39. Bogotá. 


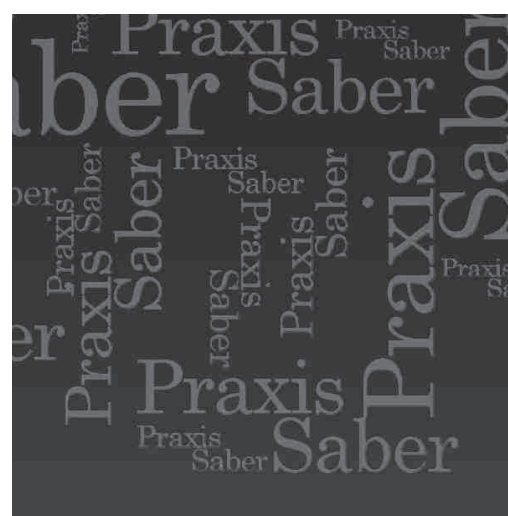

Marco Raúl Mejía Jiménez

Doctor del Proyecto interdisciplinario de Investigaciones Educativas (Chile)

Asesor Pedagógico Nacional del Programa Ondas de Colciencias. Planeta Paz. Expedición Pedagógica Nacional.

Profesor invitado Maestría en Educación

Universidad Pedagógica y

Tecnológica de Colombia marcoraulm@gmail.com

María Elena Manjarrés

Doctora en Estudios de

Desarrollo

Docente investigadora en

Pontificia Universidad Javeriana de Colombia

Coordinadora Nacional del

Programa Ondas de Colciencias

- Colombia

Artículo de Reflexión

Recibido: 2 de septiembre de 2011 Aprobado: 28 de octubre de 2011

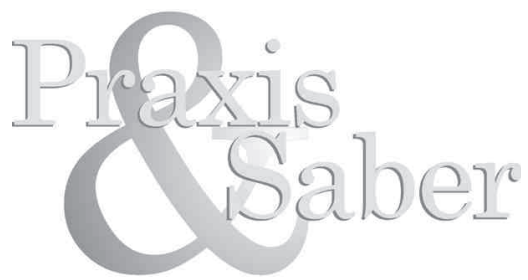

Revista de Investigación y Pedagogía Maestría en Educación. Uptc

\section{LA INVESTIGACIÓN COMO ESTRATEGIA PEDAGÓGICA UNA APUESTA POR CONSTRUIR PEDAGOGÍAS CRÍTICAS EN EL SIGLO XXI ${ }^{1}$}

\section{Resumen}

El texto busca fundar la idea de que llevar la investigación a la esfera educativa y pedagógica va a generar una reelaboración del campo de éstas, así como de los mecanismos prácticos y de acción que las han constituido. En primer lugar se realiza un rápido recorrido para reconocer la manera como se han constituido la educación y la pedagogía en la modernidad capitalista occidental; en él se observan los paradigmas, corrientes, concepciones, enfoques y líneas metodológicas y modelos, como aspectos que vertebran estas búsquedas; luego se muestra la manera como se conformó el pensamiento pedagógico en torno a la investigación, el cual da origen a diversas entradas metodológicas para ser resuelto en el cotidiano de las prácticas de los educadores; por último, se fundamenta una de esas líneas metodológicas, la de la Investigación como Estrategia Pedagógica (IEP), surgida en las construcciones de las pedagogías críticas desarrolladas en las realidades latinoamericanas y en diálogo con los enfoques surgidos y dinamizados en diferentes latitudes mundiales.

Palabras clave: Investigación pedagógica, paradigmas educativos, nuevo capitalismo, maestro.

1 Debe leerse como continuidad del texto "La Investigación como Estrategia Pedagógica”, capítulo IV del libro La reconstrucción colectiva del Programa Ondas 2006-2008. La investigación como estrategia pedagógica. Bogotá. FES-COLCIENCIASONDAS 2009. Páginas 129 a 163. 
Research as a Pedagogical Strategy

a Step Towards Critical Pedagogies for the 21st

CEnTURY

\section{Summary}

This research paper aims to promote the idea that the use of research in the fields of education and pedagogy is going to create a modification in these fields, as well as in the practical processes and actions that they have established. First of all, this paper provides a quick overview of the way in which education and pedagogy have been constituted in the Western, capitalist modern society; along with its paradigms, trends, concepts, methodological approaches, research areas and models. Next, this paper shows the way in which pedagogical thinking has been formed around research, that which gives rise to diverse methodological approaches which become the daily practice of educators. Finally, this paper closely examines one of these methodological lines, Research as a Pedagogical Strategy (Investigación como Estrategia Pedagógica - IEP), which emerged in the creation of the critical pedagogies developed in Latin American contexts, in dialogue with the emerging and dynamic approaches in different parts of the world.

Key words: Pedagogical research, educational paradigms, new capitalism, teacher.

\section{La Recherche Comme Stratégie Pédagogique un Pari Pour Construire des Pédagogies Critiques DANS LE XXIÈME SiÈCLE}

\section{Résumé}

Le texte cherche à fonder l'idée suivante : amener la recherche à la sphère éducative et pédagogique, va générer une réélaboration de leurs domaines, de même que des mécanismes pratiques et d'action que les constituent. En premier lieu, on réalise un parcours rapide pour reconnaittre la manière comme l'éducation et la pédagogie se sont constituées, dans la modernité capitaliste occidentale;dans ce parcours-là, on observe les paradigmes, courants, conceptions, approches et lignes méthodologiques et des modèles, comme des aspects qui constituent les vertèbres de ces recherches ; ensuite, on montre la manière comme la pensée pédagogique 